\title{
Influence of environmental static electric field on antioxidant enzymes activities in hepatocytes of mice
}

\author{
S.X. Wu ${ }^{1}$, Y.Q. Xu' ${ }^{1}$, G.Q. Di ${ }^{1}$, J.H. Jiang ${ }^{2}$, L. Xin ${ }^{2}$ and T.Y. Wu ${ }^{2}$ \\ ${ }^{1}$ Institute of Environmental Pollution \& Control Technology, Zhejiang University, \\ Hangzhou China \\ ${ }^{2}$ State Grid Shanghai Municipal Electric Power Company, Shanghai, China \\ Corresponding author: G.Q. Di \\ E-mail: dgq@zju.edu.cn
}

Genet. Mol. Res. 15 (3): gmr.15038800

Received May 16, 2016

Accepted June 3, 2016

Published July 25, 2016

DOI http://dx.doi.org/10.4238/gmr.15038800

Copyright $(2016$ The Authors. This is an open-access article distributed under the terms of the Creative Commons Attribution ShareAlike (CC BY-SA) 4.0 License.

\begin{abstract}
With the increasing voltage of direct current transmission line, the intensity of the environmental static electric field has also increased. Thus, whether static electric fields cause biological injury is an important question. In this study, the effects of chronic exposure to environmental static electric fields on some antioxidant enzymes activities in the hepatocytes of mice were investigated. Male Institute of Cancer Research mice were exposed for 35 days to environmental static electric fields of different electric field intensities of 9.2-21.85 $\mathrm{kV} / \mathrm{m}$ (experiment group I, EG-I), 2.3-15.4 kV/m (experiment group II, EG-II), and $0 \mathrm{kV} / \mathrm{m}$ (control group, CG). On days 7, 14, 21, and 35 of the exposure cycle, liver homogenates were obtained and the activities of antioxidant enzymes like superoxide dismutase, glutathione $S$-transferase, and glutathione peroxidase were determined, as well as the concentration of malonaldehyde. The results revealed a significant
\end{abstract}


increase in superoxide dismutase activity in both EG-I and EG-II on the 7 th $(\mathrm{P}<0.05)$ and 35 th days $(\mathrm{P}<0.01)$ of the exposure cycle compared to that in the control group. However, the other test indices such as glutathione $S$-transferase, glutathione peroxidase, and malonaldehyde showed only minimal changes during the exposure cycle. These results revealed a weak relationship between the exposure to environmental static electric fields and hepatic oxidative stress in living organisms.

Key words: Antioxidant enzymes; Environmental static electric field; Liver

\section{INTRODUCTION}

Static electric fields exist in the vicinity of high-voltage direct current transmission lines. The intensity of the static electric field generated by a $750 \mathrm{kV}$ power line can reach as much as 12 $\mathrm{kV} / \mathrm{m}$ at the cord minimal height (13 m) (Güler et al., 2006), and further increases to $21.6 \mathrm{kV} / \mathrm{m}$ when the voltage of the power line reaches $800 \mathrm{kV}$ (Chai, 2012). Both science researchers and the general population have concerned about the health effects of static electric fields.

Previous studies have focused on the biological effects of time-varying electromagnetic field. It has been demonstrated in some studies that low-frequency electromagnetic fields can cause DNA single- and double-strand breaks as well as cell apoptosis and necrosis as a result of increased free radicals induced by the time-varying fields (Ivancsits et al., 2003; Lai and Singh, 2004). Coşkun et al. (2009) exposed guinea pigs to an extremely low-frequency (ELF) electromagnetic field at $50 \mathrm{~Hz}$ and $1.5 \mathrm{mT}$ for 4 days and found a significant increase in the malonaldehyde level of the liver. Hashish et al. (2008) reported that an ELF-electromagnetic field causes physiological disturbances in mice by affecting redox balance by measuring the activities of lactate dehydrogenase, $\gamma$-glutamyl transferase, glutathione- $S$-transferase, and lipid peroxidation levels in the liver. The disturbance of free radical homeostasis in organisms is a common hypothesis for explaining the effects of ELF-electromagnetic fields (MartínezSamano et al., 2010). However, studies of the relationship between environmental static electric field exposure and oxidative stress are limited and the results are controversial (Cieslar et al., 2003, 2009; Güler et al., 2006). Thus, additional studies on the oxidative effects of environmental static electric fields are needed.

Superoxide dismutase (SOD), glutathione $S$-transferase (GST), and glutathione peroxidase (GSH-PX) are different antioxidant enzymes present in various organs and play important roles in the elimination of free radicals and protecting cells against oxidative damage. In this study, we estimated the influence of chronic exposure to environmental static electric fields on the livers of male mice by measuring the activities of these antioxidant enzymes and the concentration of malonaldehyde (MDA).

\section{MATERIAL AND METHODS}

\section{Experimental animals}

A total of 120 male ICR mice (4 weeks old, weighing $19.6 \pm 1.6 \mathrm{~g}$ ) were obtained from the Experimental Animal Center of Zhejiang Province (Hangzhou, China) and were randomly

Genetics and Molecular Research 15 (3): gmr.15038800 
divided into 3 groups: experimental group I (EG-I, $\mathrm{N}=40$ ), experimental group II (EG-II, $\mathrm{N}$ $=40$ ), and control group $(\mathrm{CG}, \mathrm{N}=40)$. Mice were housed with 10 mice per specially-made cage and were marked on their fur with picric acid to distinguish each individual. Mice were fed with standard laboratory food and had free access to water.

\section{Exposure to environmental static electric field}

ICR mice were exposed to an environmental static electric field (mainly generated by high-voltage direct current transmission lines) for 35 days in an open air environment. Autumn was chosen as the most suitable exposure season because of its optimum temperature for mouse survival. During the exposure period, the outside temperature was approximately $24.8^{\circ}$ $\pm 2.1^{\circ} \mathrm{C}$ during the daytime and $17.7^{\circ} \pm 2.6^{\circ} \mathrm{C}$ at night. The experimental and control groups were settled in 3 different locations under the same environmental conditions except the intensities of static electric field. EG-I was exposed to a static electric field with an intensity of 9.2-21.85 kV/m, EG-II was exposed to a relatively low electric field with an intensity of 2.3$15.4 \mathrm{kV} / \mathrm{m}$, and the static electric field intensity in the place where CG was placed was near 0 $\mathrm{kV} / \mathrm{m}$. The overlapping intensities of EG-I and EG-II were mainly caused by different weather conditions. However, the relative exposure intensity of EG-I and EG-II at the same time was certain, and the exposure intensity of EG-I was always higher than that of EG-II (EG-II was further away from the high-voltage direct current transmission line than EG-I). Therefore, the exposure dose of EG-I was higher than that of EG-II.

All groups were exposed for $24 \mathrm{~h}$ each day except days on which it was raining. The actual exposure time and actual intensity of the environmental static electric field under different weather conditions was measured and recorded.

\section{Estimation of antioxidant enzyme activity}

On the 7th, 14th, 21st, and 35th days of the exposure cycle, some animals from each group (10 mice per group) were sacrificed under ether anesthesia. Their livers were obtained and weighed immediately. Next, $10 \%$ liver homogenates were prepared in 9 volumes of $0.86 \%$ saline solution using a homogenizer (Tissuelyser-48, Shanghai, China). The activities of antioxidant enzymes including SOD, GSH-PX, and GST, as well as the concentration of MDA, were tested using reagent kits purchased from Nanjing Jiancheng Bioengineering Institute (Nanjing, China). SOD activity was evaluated based on the ability of SOD to inhibit the reduction of nitroblue tetrazolium by superoxide at $560 \mathrm{~nm}$. GSH-PX activity was determined by enzyme reaction rate at which GSH-PX could catalyze the oxidation of reduced glutathione (GSH) to oxidized glutathione in the presence of $\mathrm{H}_{2} \mathrm{O}_{2}$. GST activity was quantified by measuring the ability of GSH to catalyze the substrate reaction of 2,4-dinitrochlorobenzene with GSH. MDA was measured using the thiobarbituric acid assay, which is based on the MDA reaction with thiobarbituric acid to give thiobarbituric acid reactive substances, a red species that absorbs at $535 \mathrm{~nm}$. The catalog numbers of the kits from the Nanjing Jiancheng Bioengineering Institute were A001, A005, A004, and A003, respectively. The results of these assays were normalized to the total amount of protein as measured using the bicinchoninic acid method. Before testing, preliminary experiments were conducted to determine optimal sample amounts. All animal experiments were performed in accordance with the Quality Management Approach to Laboratory Animals, and all efforts were made to minimize the number of animals used and their suffering.

Genetics and Molecular Research 15 (3): gmr.15038800 


\section{Statistical analysis}

All results are reported as mean values \pm SD for each group. Comparisons between different groups were performed by one-way analysis of variance followed by appropriate post-hoc tests including multiple comparison tests, using Fisher least significant difference tests when the variance was equal and using Tamhane tests when the variance was unequal. All comparisons were carried out using SPSS 20.0 (SPSS, Inc., Chicago, IL, USA). Differences were considered significant at $\mathrm{P}<0.05$ (with an asterisk representing $\mathrm{P}<0.05$ and two asterisks representing $\mathrm{P}<0.01$ in following figures).

\section{RESULTS}

\section{SOD activity in hepatocytes}

The mean values of SOD activity in the hepatocytes of mice from control and exposed groups ( $\mathrm{N}=10$ in each group) on different days of the exposure cycle are presented in Figure 1. There was a significant increase $(\mathrm{P}<0.05)$ in SOD activity in both EG-I and EG-II on the 7th day of exposure compared with that in the CG. Additionally, the SOD activity statistically increased in both EG-I $(\mathrm{P}<0.01)$ and EG-II $(\mathrm{P}<0.01)$ on the 35th day of exposure. However, the hepatic SOD activities of mice exposed to the environmental static electric field for 14 and 21 days showed no significant difference between EG and CG.

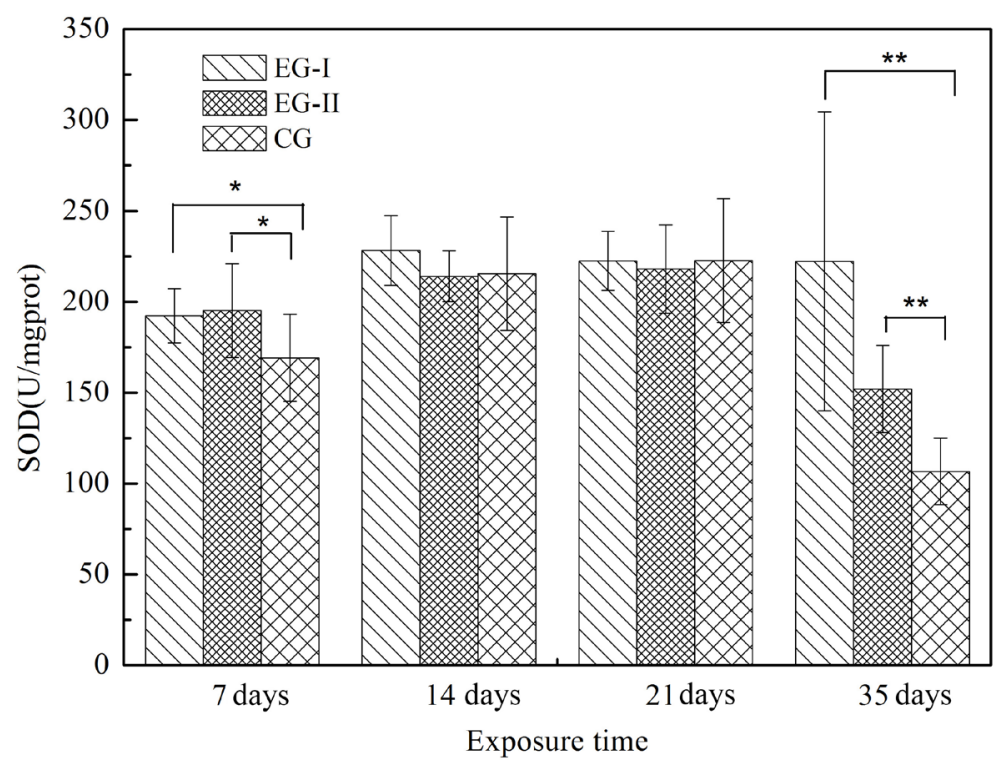

Figure 1. Mean values of SOD activity in hepatocytes of mice from control and differentially exposed groups $(\mathrm{N}=$ 10 in each group) on particular days of the exposure cycle.

\section{GST activity in hepatocytes}

The mean values of GST activity in hepatocytes of mice from control and exposed

Genetics and Molecular Research 15 (3): gmr.15038800 
groups ( $\mathrm{N}=10$ in each group) on different days of the exposure cycle are presented in Figure 2. On the 35th day of exposure, hepatic GST activity in EG-I was higher than that in EGII, and the levels were both higher than that in the CG; however, the difference was not significant. Additionally, there were no significant differences in GST activity in EG-I and EG-II compared with CG on the 7th, 14th, 21st, and 35th days of exposure.

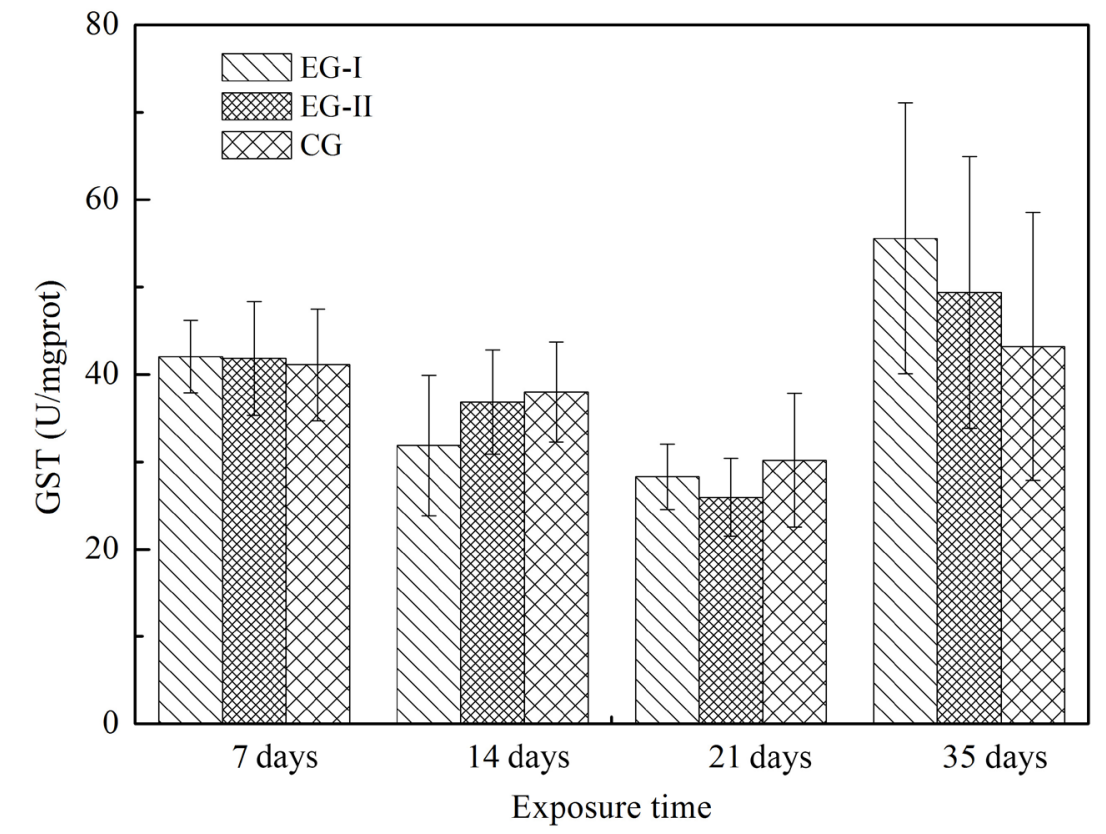

Figure 2. Mean values of GST activity in hepatocytes of mice from control and differentially exposed groups $(\mathrm{N}=$ 10 in each group) on particular days of the exposure cycle.

\section{GSH-PX activity in hepatocytes}

The mean values of GSH-PX activity in hepatocytes of mice from control and exposed groups ( $\mathrm{N}=10$ in each group) on different days of the exposure cycle are presented in Figure 3. Hepatic GSH-PX activity in EG-I was higher than that in CG at all exposure times; however, the difference was not significant. Additionally, there were no significant differences in GSH-PX activity in EG-II compared with that in the CG on the 7th, 14th, 21st, and 35th days of exposure.

\section{Concentration of MDA in hepatocytes}

The mean values of MDA concentration in hepatocytes of mice in control and exposed groups ( $\mathrm{N}=10$ in each group) on different days of the exposure cycle are presented in Figure 4. As exposure time increased, MDA concentration increased first and then decreased in each group. However, no statistically significant changes were observed among these three groups on each day of the exposure cycle. 


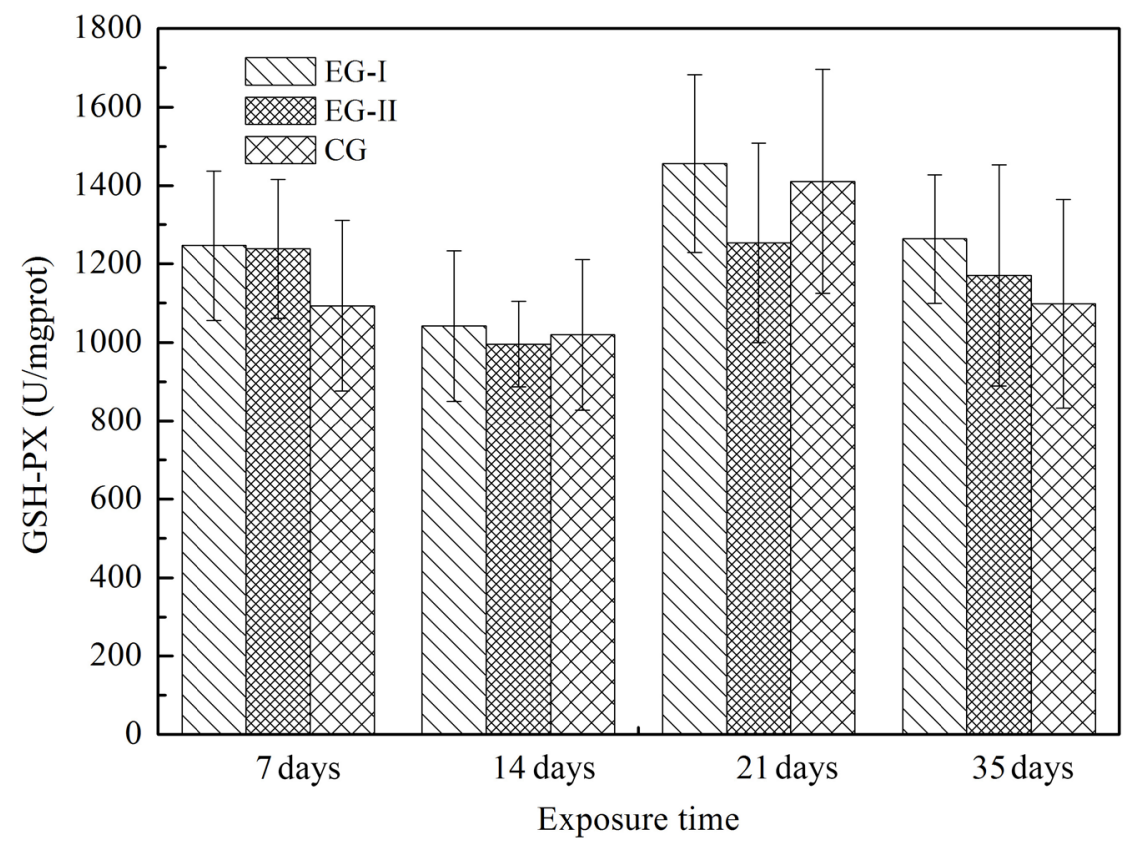

Figure 3. Mean values of GSH-PX activity in hepatocytes of mice from control and differentially exposed groups $(\mathrm{N}=10$ in each group) on particular days of the exposure cycle.

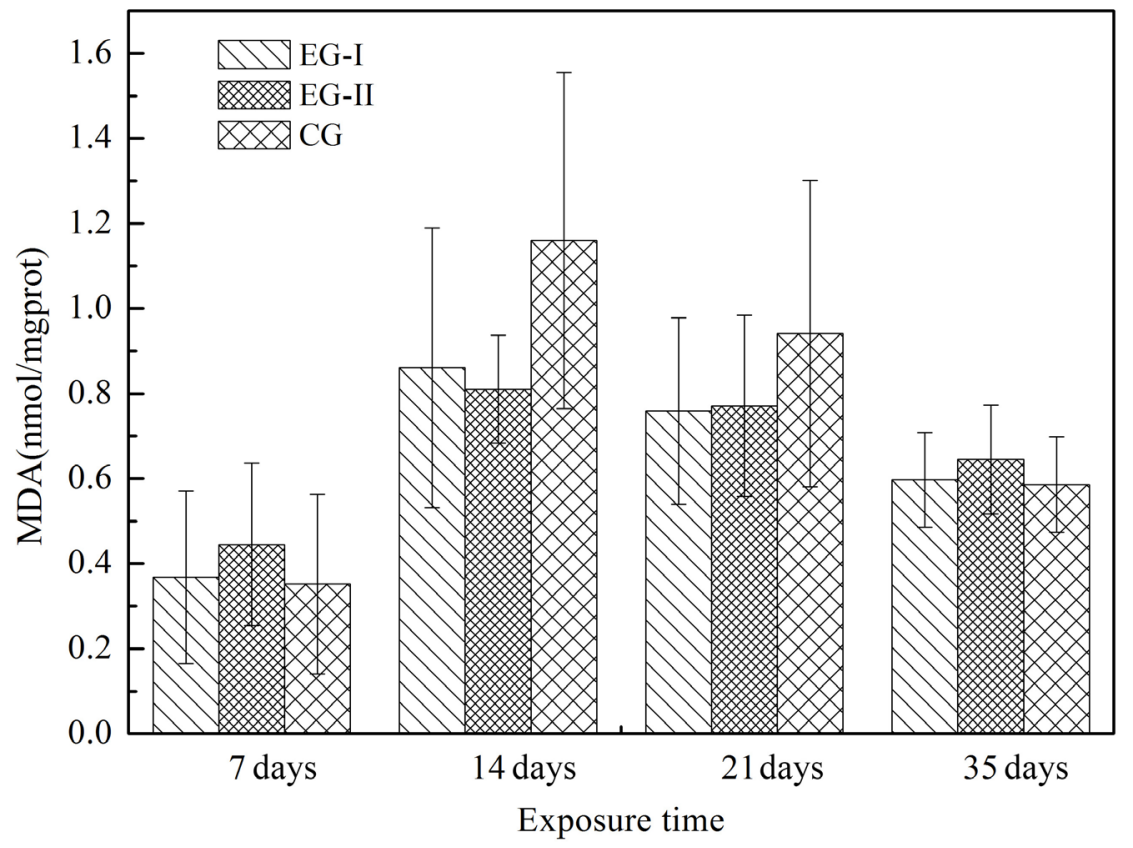

Figure 4. Mean values of the concentration of MDA in hepatocytes of mice from control and differentially exposed groups ( $\mathrm{N}=10$ in each group) on particular days of the exposure cycle. 


\section{DISCUSSION}

Free radicals, which are considered to be the main cause of disease and aging, are a class of highly reactive molecules that attack biomacromolecules and destroy body cells. Under normal conditions, the production and removal of free radicals inside humans are in homeostasis. However, excessive free radicals can be produced when humans are under stress conditions. Excess free radicals can cause serious oxidative damage of many important biological macromolecules and lead to cell damage. Free radical scavengers, which can be divided into two major categories of antioxidant enzymes and antioxidants, are a group of substances that can prevent and repair damage inflicted by these free radicals. GSH is a powerful antioxidant that plays an important role in the antioxidant defense system in humans. It is mainly produced by the liver and then transported to other parts of the organism. GSH not only eliminates free radicals by directly reacting with a variety of reactive oxygen species, but also combines and removes some organic peroxides catalyzed by GSH-PX and GST. Thus, GSH-PX and GST are considered to be sensitive markers of free radical contents and oxidative reaction intensity. Cieslar et al. (2003) exposed male Wistar rats to electric fields with intensities of 16,32 , and $48 \mathrm{kV} / \mathrm{m}$ for 10 days ( $4 \mathrm{~h}$ daily) and found that the activities of glutathione reductase and glutathione peroxidase in erythrocytes was significantly increased in rats exposed to the electric field with the highest intensity of $48 \mathrm{kV} / \mathrm{m}$ compared to in control rats. In our study, the activities of GSH-PX and GST were measured, and no differences were observed between the exposure groups and the CG. As a result, environmental static electric fields with intensities of no higher than $22 \mathrm{kV} / \mathrm{m}$ have minimal adverse effects on oxidative stress in the liver based on glutathione reductase and GSH-PX activities.

SOD is another antioxidant enzyme that functions with GSH-PX and GST to protect organisms from toxic free radicals, mainly superoxide anion free radicals. The liver is the most important detoxification organ in the body and is abundant in the mitochondria, the main organelle generating superoxide anion free radicals (Irmak et al., 2002). Thus, estimating the hepatic activity of SOD reflects the oxidative stress state to some extent. In this study, statistically significant increases in the hepatic activity of SOD were found in EG-I and EGII after exposure times of 7 and 35 days as compared to that in the CG. The increase in SOD activity may be regarded as an indicator of increased superoxide anion free radical production after different exposure times. Güler et al. (2006) hypothesized that molecular $\mathrm{O}_{2}$ was transformed to superoxide anion free radicals as a result of energy transfer in the tissue in an electric field. Thus, during the early exposure in our study, enhanced production of superoxide anion free radicals may have increased SOD synthesis, which can eliminate superoxide anion free radicals by forming $\mathrm{H}_{2} \mathrm{O}_{2}$. This may be a self-regulatory mechanism that protects cells from injury caused by increased reactive oxygen species. This hypothesis is supported by the similar activity of SOD between the exposure group and the CG $(\mathrm{P}>0.05)$ in the later exposure period (14 and 21 days). However, SOD activity was again significantly increased in both exposure groups with increasing exposure time ( 35 days). This may be because the adverse effect induced by the environmental static field was cumulative after the long-time exposure and the organism was not able to regulate itself when the adverse effect exceeded a certain threshold level. The SOD activity of CG measured on 35 day was much lower than in the other groups, which may have been influenced by mouse growth. However, further studies are required. Cieslar et al. (2003) exposed male Wistar rats to a static electric field with an intensity of $32 \mathrm{kV} / \mathrm{m}$ for 10 days $(4 \mathrm{~h} /$ day) and found no significant differences in

Genetics and Molecular Research 15 (3): gmr.15038800 
erythrocytes or serum SOD activity, which is similar to our findings. In addition, the difference became significant $(\mathrm{P}<0.05)$ in the serum when the exposure level was increased to $48 \mathrm{kV} / \mathrm{m}$, indicating that the effect is dose-dependent.

MDA concentrations in hepatocytes were also determined in this study. MDA is a specific and chemically stable product of lipid peroxidation, which is the process during which free radicals attack polyunsaturated fatty acids in cell membranes, resulting in cell damage. Thus, MDA is also regarded as a reliable biological marker of oxidative stress (Güler et al., 2006). It was reported that the concentration of MDA in the homogenates of liver tissue showed no significant differences between groups of electric field-exposed rats $(25 \mathrm{kV} / \mathrm{m}$, 8 h daily for 56 consecutive days) and sham-exposed rats (Cieslar et al., 2009). The results of this study agree with those of previous studies showing insignificant differences in MDA concentrations after different exposure periods. These data indicate that static electric fields of certain intensities do not increase lipid peroxidation in biological membranes in liver tissues.

\section{CONCLUSIONS}

The influence of exposure to environmental static electric fields with intensities of 9.2-21.85 kV/m (EG-I) and 2.3-15.4 kV (EG-II) for 35 days on oxidative stress in the liver of mice was investigated in this study. The results showed a significant increase in hepatic SOD activity in both EG-I and EG-II on the 7th $(\mathrm{P}<0.05)$ and 35th days $(\mathrm{P}<0.01)$ of exposure cycle compared to that in the CG. There were no differences in hepatic GST activity, GSH-PX activity, and MDA concentration between groups. These results indicate that the environmental static electric field in this study did not cause serious liver hazards for living organisms. There was only a weak relationship between exposure to the environmental static electric field and hepatic oxidative stress. This likely stimulated antioxidant reactions in the liver by producing more superoxide anion free radicals. However, the adverse effect was not persistent and could be alleviated by the body's own regulation mechanisms to some extent.

\section{Conflicts of interest}

The authors declare no conflict of interest.

\section{ACKNOWLEDGMENTS}

Research supported by the Science and Technology Funds from the State Grid Corporation of China (Contract \#SGTYHT/14-JS-188).

\section{REFERENCES}

Chai XD (2012). Analysis of Electric Field under Wires and Corona Loss about UHVDC Power Transmission Lines. Master's thesis, Chongqing University, Chongqing, China.

Cieslar G, Sowa P and Sieron A (2003). Influence of high voltage static electric field on antioxidant enzymes activity in male rats. Proceedings of the 25th Annual International Conference of the IEEE Engineering in Medicine and Biology Society, Mexico, 3257-3260.

Cieslar G, Fiolka J, Mrowiec J, Sowa P, et al. (2009). Effect of Exposure to Static, High Voltage Electric Field Generated Nearby HVDC Transmission Lines on Antioxidant Activity of Hepatocytes in Rats. PIERS Proceedings, Moscow, Russia, 1092-1096.

Genetics and Molecular Research 15 (3): gmr.15038800 
Coşkun S, Balabanli B, Canseven A and Seyhan N (2009). Effects of continuous and intermittent magnetic fields on oxidative parameters in vivo. Neurochem. Res. 34: 238-243. http://dx.doi.org/10.1007/s11064-008-9760-3

Güler G, Seyhan N and Aricioğlu A (2006). Effects of static and $50 \mathrm{~Hz}$ alternating electric fields on superoxide dismutase activity and TBARS levels in guinea pigs. Gen. Physiol. Biophys. 25: 177-193.

Hashish AH, El-Missiry MA, Abdelkader HI and Abou-Saleh RH (2008). Assessment of biological changes of continuous whole body exposure to static magnetic field and extremely low frequency electromagnetic fields in mice. Ecotoxicol. Environ. Saf. 71: 895-902. http://dx.doi.org/10.1016/j.ecoenv.2007.10.002

Irmak MK, Fadillioğlu E, Güleç M, Erdoğan H, et al. (2002). Effects of electromagnetic radiation from a cellular telephone on the oxidant and antioxidant levels in rabbits. Cell Biochem. Funct. 20: 279-283. http://dx.doi.org/10.1002/cbf.976

Ivancsits S, Diem E, Jahn O and Rüdiger HW (2003). Intermittent extremely low frequency electromagnetic fields cause DNA damage in a dose-dependent way. Int. Arch. Occup. Environ. Health 76: 431-436. http://dx.doi.org/10.1007/ $\underline{\mathrm{s} 00420-003-0446-5}$

Lai H and Singh NP (2004). Magnetic-field-induced DNA strand breaks in brain cells of the rat. Environ. Health Perspect. 112: 687-694. http://dx.doi.org/10.1289/ehp.6355

Martínez-Sámano J, Torres-Durán PV, Juárez-Oropeza MA, Elías-Viñas D, et al. (2010). Effects of acute electromagnetic field exposure and movement restraint on antioxidant system in liver, heart, kidney and plasma of Wistar rats: a preliminary report. Int. J. Radiat. Biol. 86: 1088-1094. http://dx.doi.org/10.3109/09553002.2010.501841

Genetics and Molecular Research 15 (3): gmr.15038800 